\title{
Image Pre-compensation: Balancing Contrast and Ringing
}

\author{
$\mathrm{Yu} \mathrm{Ji}^{1} \quad$ Jinwei $\mathrm{Ye}^{1} \quad$ Sing Bing Kang ${ }^{2} \quad$ Jingyi $\mathrm{Yu}^{1}$ \\ ${ }^{1}$ University of Delaware, Newark, DE, USA. \{yuji, jye, yu\}eeecis.udel.edu \\ ${ }^{2}$ Microsoft Research, Redmond, WA, USA. sbkangemicrosoft.com
}

\begin{abstract}
The goal of image pre-compensation is to process an image such that after being convolved with a known kernel, will appear close to the sharp reference image. In a practical setting, the pre-compensated image has significantly higher dynamic range than the latent image. As a result, some form of tone mapping is needed. In this paper, we show how global tone mapping functions affect contrast and ringing in image pre-compensation. In particular, we show that linear tone mapping eliminates ringing but incurs severe contrast loss, while non-linear tone mapping functions such as Gamma curves slightly enhances contrast but introduces ringing. To enable quantitative analysis, we design new metrics to measure the contrast of an image with ringing. Specifically, we set out to find its "equivalent ringing-free" image that matches its intensity histogram and uses its contrast as the measure. We illustrate our approach on projector defocus compensation and visual acuity enhancement. Compared with the state-of-the-art, our approach significantly improves the contrast. We believe our technique is the first to analytically trade-off between contrast and ringing.
\end{abstract}

\section{Introduction}

All projectors introduce some form of visual blurring due to its optics and possibly non-planar projection surface. One way to reduce this problem is to first characterize its blur (defocus) kernel and preprocess the image such that the resulting projected image is sharp. The preprocessing step is called image pre-compensation.

More generally, image pre-compensation is a long standing problem in image processing with numerous applications in computer vision and graphics. Given a sharp reference image $I$ and the blur kernel or point spread function (PSF) $K$, the goal is to find a "compensated" image $J$ which, after being convolved with $K$, will appear close to $I$. In the projection defocus compensation example above, by projecting $J$, the actual perceived image should appear nearly focused.
Pre-compensation vs. Deblurring. At first glance, image pre-compensation may resemble image deblurring as both can be viewed as "deconvolution". The two problems, however, are inherently different. In image deblurring, there always exist some "ground truth" $J$ to produce $I$ under kernel $K$. The ill-posedness of deblurring rises from the invertibility of $K$, i.e., if $K$ is not invertible, there exist multiple $J$ that can produce the same $I$. To resolve this ambiguity, classical Wiener filter [25] uses regularization to enforce invertibility whereas more advanced solutions add priors such as image statistics, e.g., gradient sparsity $[14,16,13,15]$, edge sharpness $[10,11,4]$ or new irradiance-based blur model [3] to constrain the solution.

In image pre-compensation, there usually does not exist any valid solution $J$. In projector defocus compensation for example, the convolution kernel is a low pass filter that removes the high frequency components of $J$. $J$ is expected to preserve sharpness even after being blurred. Therefore, the problem is ill-posed in that no "ground truth" solution exists.

Dynamic Range Problem. A serious problem in image pre-compensation is the significant increase of dynamic range. Assume $K$ is invertible, conceptually $J$ can be directly computed as

$$
J=I \otimes K^{-1}
$$

where $\otimes$ denotes convolution. Consider a randomly generated 1D invertible kernel and a 1D image of a step edge $[0,1]^{1}$. The resulting $J$ has range $[-2.9,4.9]$, as shown in Fig. 1. In the projector defocus compensation case, we will need to use $J$ as the input to the projector and therefore we will have to first compress the rang (tone map) $J$ to $[0,1]$.

The simplest tone mapping function is linear range compression. In the simple 1D example above, if we apply linear tone mapping on $J$ as $l(J)$, the resulting $I=l(J) \otimes K$ will have dynamic range $[0.4,0.6]$, much narrower than its original one $[0,1]$, as shown in Fig. 1 . In the projector defocus case, it will lead to severe contrast loss. More sophisticated tone mapping such as Gamma function slightly

\footnotetext{
${ }^{1}$ In this paper, we assume the display's dynamic range is $[0,1]$
} 


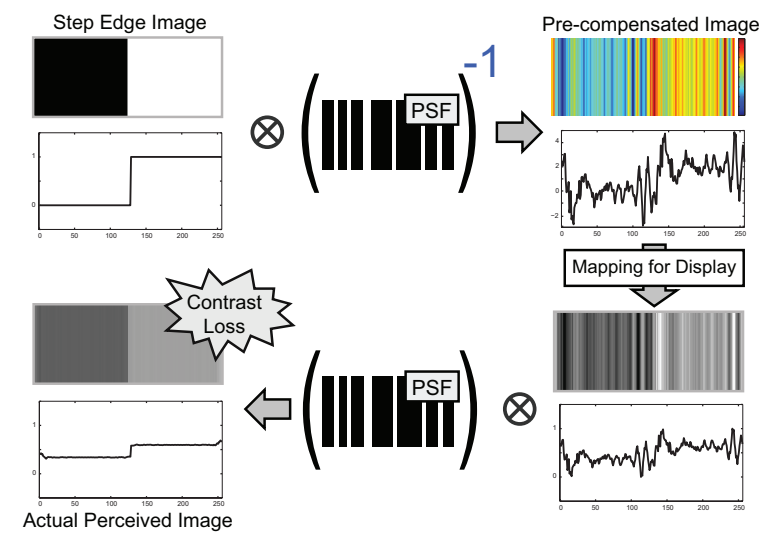

Figure 1. The pre-compensated image of a step edge under an invertible kernel incurs dynamic range burst. Linear tone mapping produces ringing-free result but significantly reduces the scale (contrast) of the step.

broadens the dynamic range and contrast of $I$ but at at the same time introduces ringing. Most previous approaches assume relatively small kernels $K$ and resort to optimization schemes such as steepest descent [26] and Wiener filters $[2,19]$. However, these techniques are less effective on larger kernels. By far, only a handful of techniques address the role of tone mapping functions in image deconvolution/deblurring $[12,3,23]$ while the analysis in image precompensation is largely missing.

Our paper is the first to systematically study how the tone mapping function affects ringing and contrast in image pre-compensation. In this work, we focus on global tone mapping functions. We first show that linear tone mapping completely eliminates ringing but incurs severe contrast loss. In contrast, non-linear tone mapping functions such as Gamma curves slightly enhances contrast but introduces ringing. To conduct a quantitative analysis, we design new metrics to measure the contrast of an image with ringing. Specifically, we set out to find its "equivalent ringing-free" image that matches the intensity histogram and uses its contrast as the measure. Our approach hence enables reliable comparisons between different tone mapping functions as well as effective constructions of specific tone mapping functions to achieve a target contrast.

We demonstrate our technique in a range of applications. For projector defocusing, we show that our technique outperforms the state-of-the-art solutions based on steepest descent [26] and Wiener filters [2, 19] on large kernels. For visual acuity enhancement, our technique can improve the visual experience for people with myopia or hyperopia when not wearing corrective lenses. Specifically, we provide a simple user interface to trade between ringing and contrast that allow user to adjust the ringing control factor for generating the optimal curve. Our user study shows that our technique is effective and comparable to the state-ofthe-art solutions [1,9] in both visual quality and quantitative measures.

\section{Tone Mapping in Image Pre-compensation}

We first study how tone mapping affects the dynamic range, contrast, and ringing in image pre-compensation. The phenomena of significant dynamic range stretch in image deconvolution has been widely documented in signal processing [22] and computer graphics/vision literature $[26,1,8,9]$. To briefly reiterate, the stretch is due to matrix inversion. Recall that the convolution kernel $K$ can be written in form of a Toeplitz matrix with blockcirculant-with-circulant-block (BCCB) structure which can be diagonalized by singular value decomposition (SVD) as $K=U \Lambda V^{*}$, where $U$ and $V$ are the left and right singular vectors and $\Lambda$ is a diagonal matrix composed of the square roots of eigen values. Assume $\lambda_{\min }$ is minimum eigen value in $\Lambda$. Since the eigen values of $K^{-1}$ are the reciprocal of $K$ 's, the maximum in $\Lambda^{\prime}$ is then $\lambda_{\max }^{\prime}=1 / \lambda_{\min }$. If $\lambda_{\min }$ is close to zero, $\lambda_{\max }^{\prime}$ can be very large, resulting range expansion in $J$. This implies that $J$ can be of a much higher dynamic range (HDR) and therefore cannot be "physically" implemented, e.g., used as an input to the projector. The question is then how to map dynamic range of $J$ to $[0,1]$.

Before proceeding, we clarify our notations. Assume the sharp reference image $I$ has range $[0,1]$ and the computed $J$ has range $[\min (J), \max (J)]$. Given a tone mapping function $f:[\min (J), \max (J)] \mapsto[0,1]$, we map $J$ to $J_{f}$ as the final pre-compensated image and denote the resulting convolution result as $I_{f}=J_{f} \otimes K=f(J) \otimes K$. An ideal $f$ should produce $I_{f} \approx I$.

\subsection{Linear Mapping: The Baseline Performance}

As shown in the example of Fig. 1, the simplest $f$ is the linear compression function $l$ :

$$
J_{l}=l(J)=\frac{J-\min (J)}{r}
$$

where $r=\max (J)-\min (J)$, i.e., the span of the dynamic range. Convolving $J_{l}$ with $K$, we have $I_{l}$ as

$$
I_{l}=l(J) \otimes K=\frac{(I-\mu)}{r}
$$

where $\mu=\min (J) \otimes K$ is a constant. $I_{l}$ hence shifts and scale $I$ and therefore should be ringing free. However, it suffers from significant contrast loss.

For a ringing free image $I$, we can define its contrast by root mean square (RMS) as:

$$
c(I)=\sqrt{\frac{1}{n} \sum_{i=1}^{n}(I(i)-\bar{I})^{2}}
$$

where $n$ is number of pixels and $\bar{I}$ is the average intensity value. 
Since $I_{l}$ is ringing free, we can compute its contrast factor $\zeta$ with respect to $I$ as $\zeta\left(I_{l}\right)=c\left(I_{l}\right) / c(I)=1 / r$. Notice that if $I$ is of uniform intensity, $c(I)=c\left(I_{f}\right)=0$. In this singular case, we define $\zeta\left(I_{f}\right)=1$, indicating no contrast lost. Notice that $r$ can be very large even with a moderate size $K$. For example, a $5 \times 5$ Gaussian kernel of $\sigma=2.5$ (here we assume $J$ can be obtained by Wiener filter) will result in $r=17$, i.e., the contrast loss is significant $(\zeta=1 / 17)$. In this paper, we use $J_{l}$ as the baseline result and compare it with other tone mapping functions.

\subsection{General Tone Mapping}

For a general tone mapping function, we assume it is constructed by composing an additional tone mapping function $f:[0,1] \mapsto[0,1]$ onto the baseline result $J_{l}$. This significantly simplifies our analysis. For example, many classical tone mapping functions such as Gamma curves can be directly modeled using $f$. The final tone mapping function hence is $f \circ l=f(l(J))=f\left(J_{l}\right)$ and we denote $J_{f}$ as the pre-compensated result and $I_{f}$ the perceived image.

General Linear Mapping. Let us first consider the general linear mapping with truncation:

$$
J_{f}= \begin{cases}0, & 0 \leq J_{l}<-\frac{b}{m} \\ m J_{l}+b, & -\frac{b}{m} \leq J_{l} \leq \frac{1-b}{m} \\ 1, & \frac{1-b}{m}<J_{l} \leq 1\end{cases}
$$

When no truncation occurs, we have

$$
I_{f}=\left(m J_{l}+b\right) \otimes K=m I_{l}+\delta
$$

where $\delta=b \otimes K$ is a constant. Similar to the baseline case, $I_{f}$ is also ringing-free and its contrast factor is

$$
\zeta\left(I_{f}\right)=\frac{c\left(I_{f}\right)}{c(I)}=\frac{c\left(I_{f}\right)}{r \cdot c\left(I_{l}\right)}=\frac{m}{r}
$$

$m=1$ corresponds to the baseline function $l$. If $m<1$, $c\left(I_{f}\right)<c\left(I_{l}\right)$, i.e., we will lose more contrast. If $m>1$, $c\left(I_{f}\right)>c\left(I_{l}\right)$, we will gain contrast. However, when $m>$ 1 , many pixels in $J_{f}$ will saturate and need to be clamped to 0 or 1 . As a result, although the contrast is enhanced, $I_{f}$ will be contaminated by clamping. We denote the general linear mapping without truncation as $f_{m}$ ( $m$ is the slope) and we will use it to model the contrast on ringing-corrupted images.

Non-linear Mapping and Ringing. When $f$ is nonlinear, $I_{f}$ will exhibit the ringing artifacts. The cause of ringing can be explained in the frequency domain. Assume the PSF $K$ at a specific frequency $\omega_{n}$ is $a_{n}$. Therefore, the corresponding coefficient of $K^{-1}$ at frequency $\omega_{n}$ is $1 / a_{n}$. Let $I$ be a step edge function and its corresponding Fourier coefficient at $\omega_{n}$ is $v / n$ for $n \neq 0$, where $v$ is some
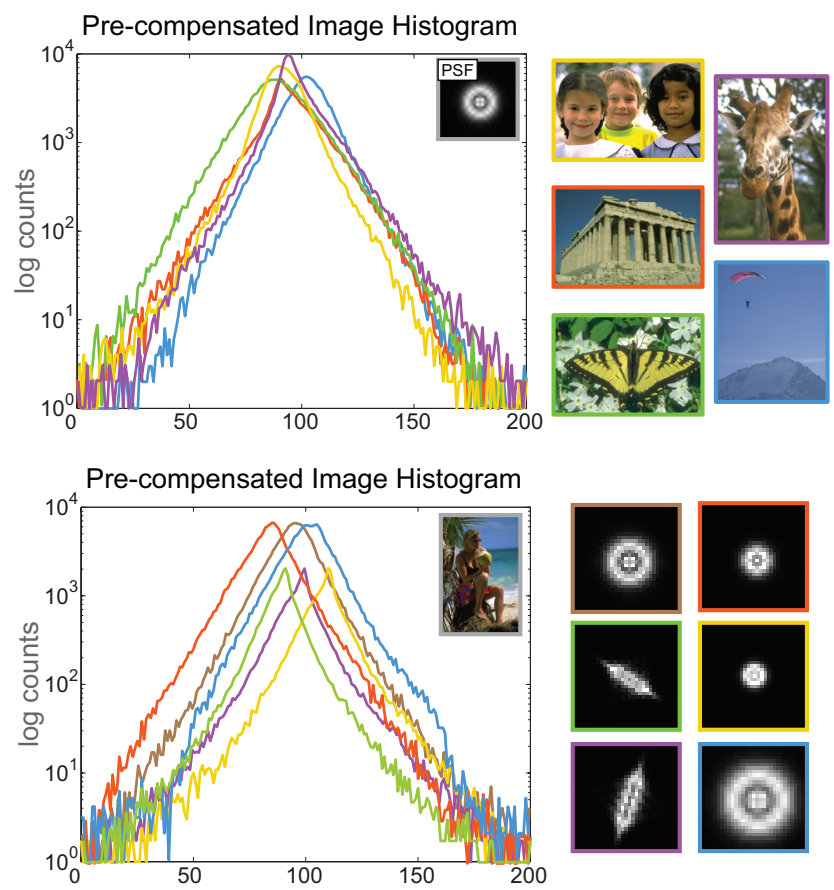

Figure 2. Histograms of pre-compensated natural images. Top: the histograms of 5 natural images from BSDS500 [17] precompensated by an invertible kernel. Bottom: the histogram of a fixed image pre-compensated by 6 different kernels.

constant. By Eqn 1, the coefficient of $J$ at frequency $\omega_{n}$ is $v / n \cdot 1 / a_{n}$.

If $f$ is a linear function, the coefficient of $I_{f}$ at frequency $\omega_{n}$ will be $\kappa \cdot v / n \cdot 1 / a_{n} \cdot a n=\kappa v / n$, where $\kappa$ is a constant scaling factor introduced by $f$. Therefore, the spectrum of $I_{f}$ will be a scaled version of $I$, i.e., $I_{f}$ will be contrast reduced step edge function and there will be no ringing artifacts, which is consistent with our conclusion in the linear case.

If $f$ is a non-linear, Farid [5] proved using Taylor's series that the coefficients at frequency $\omega_{n}$ for $J_{f}$ will be scaled non-linearly and non-uniformly, i.e., it will no longer be a scaled version of $v / n \cdot 1 / a_{n}$ and convolving it with $K$ will not cancel out $a_{n}$. As a result, $I_{f}$ will no longer be a step edge function but a signal corrupted by non-uniformly scaled high frequencies. Visually, it will exhibit strong ringing artifacts. Similar analysis has been carried out in [23].

\subsection{Disambiguating Contrast from Ringing}

The existence of ringing poses significant difficulty in measuring contrast. Since ringings appear as oscillating patterns, we cannot directly apply Eqn. 4 as the contrast measure. Specifically, a low contrast image with severe ringing can still produce large RMS contrast (Eqn. 4). In principle, the contrast of a gray-level image should not be modified by ringing, since this artifact does not introduce 


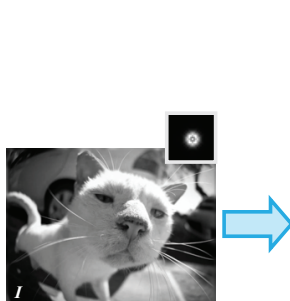

(a)

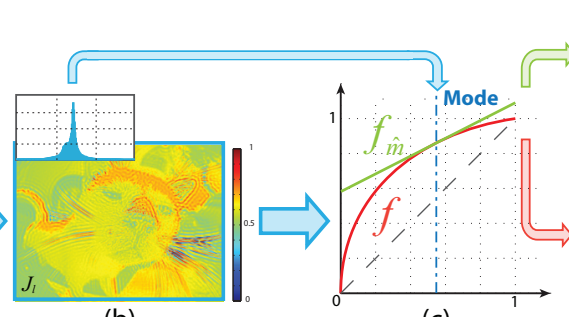

(b) (c)

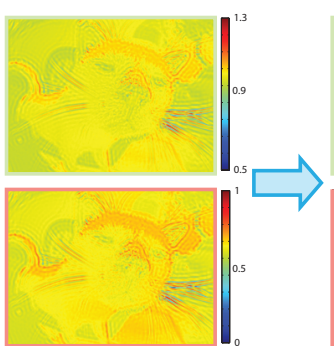

(d)

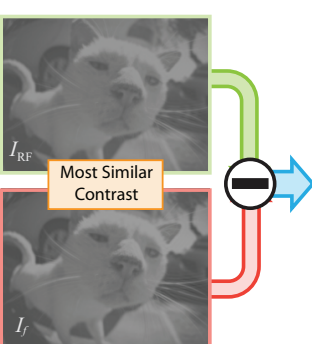

(e)

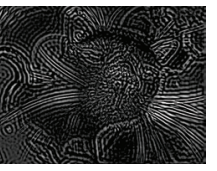

(f)

Figure 3. Measuring contrast and ringing under non-linear tone mapping $f$. (a) The sharp reference image $I$ and kernel $K$; (b) The baseline pre-compensated image $J_{l}$ and its histogram $H\left(J_{l}\right)$; (c) We compute the tangent line $f_{\hat{m}}$ of $f$ at the mode of $H\left(J_{l}\right)$; (d) Linear tone mapping using $m$ produces ringing free result $I_{\mathrm{RF}}$ whereas $f$ produces ringing-corrupted result $I_{f}$; (e) The contrast of $I_{f}$ is nearly the same as the contrast of $I_{\mathrm{RF}}$; (f) $\left|I_{\mathrm{RF}}-I_{f}\right|$ approximates the ringing in $I_{f}$.

additional meaningful content. However, the RMS contrast will be artificially boosted due to intensity variance. Alternative contrast definitions such as Weber contrast or Michelson contrast [21] that consider the minimum and maximum luminance are not able to disambiguate actual contrast from ringing. We are not aware of work done on measuring contrast under ringing.

Recall that the term "contrast" should characterize the group behavior of pixels. We consider the intensity histograms of the baseline pre-compensated result $J_{l}$ and the general tone mapped result $J_{f}$. Our key observation here is that $J_{l}$ contains ringing due the Gibbs phenomenon by deconvolution. Further, the deconvolution behaves as derivatives of a natural image, therefore the intensity histogram $H\left(J_{l}\right)$ follows the Laplacian distribution. In Fig. 2(a), we randomly select 5 images from the BSDS500 database [17] and compute their pre-compensated images using a fixed kernel. In Fig. 2(b), we fix the image but apply 6 different kernels to pre-compensate the image. The intensity histograms of the resulting pre-conditioned images consistently follow the Laplacian distribution. More validations are included in the supplementary materials.

Next, let us consider how a tone mapping function $f$ transforms $H\left(J_{l}\right)$. In the linear function $f_{m}$, the offset $b$ shifts the histogram whereas the slope $m$ stretches it. In the non-linear case, we can conduct a first order approximation to $f$ by using the tangent line at the mode of $H\left(J_{l}\right)$ (i.e., the most frequent intensity). This leads to a new contrast factor measure under an arbitrary tone mapping function $f$ : we first compute $H\left(J_{l}\right)$ and locate mode $\hat{J}_{l}$; next, we compute the tangent line $f_{\hat{m}}$ on $f$ at point $\hat{J}_{l}$; finally, we use $f_{\hat{m}}$ to linearly tone map $J_{l}$ to $J_{f_{\hat{m}}}$.

Since $f_{\hat{m}}$ is linear, $I_{f_{\hat{m}}}=J_{f_{\hat{m}}} \otimes K$ is ringing free. We call $I_{f_{\hat{m}}}$ the "equivalent ringing free" image of $I_{f}$ and denote it as $I_{\mathrm{RF}}$. We then compute the contrast of $I_{\mathrm{RF}}$ using Eqn. 4 and treat it as the contrast of $I_{f}$. Moreover, computing $I_{\mathrm{RF}}$ has another usage: we now can quantitatively measure ringing of $I_{f}$ as $\Gamma=\left|I_{f}-I_{\mathrm{RF}}\right|$. This is consistent with the observation that ringing depends on both the input pre-compensated image $J_{l}$ (by which we locate the mode of $H\left(J_{l}\right)$ ) and the tone mapping function $f$ (by which we compute the tangent function at the mode). Fig. 3 shows the complete pipeline for measuring the contrast and ringing under an arbitrary $f$.

\section{Tone Mapping Function Selection}

Our quantitative measures of contrast and ringing enables reliable comparisons between various tone mapping functions and feasible constructions of tone mapping function to achieve specific contrast.

\subsection{Contrast-Priority Tone Mapping}

We first show how to construct a tone mapping function to achieve a specific contrast. Given a desired contrast factor $\zeta$, we can directly compute the slope $m$ of the corresponding linear mapping function using Eqn. 7 as $m=r \cdot \zeta$. Since $\zeta$ is expected to outperform the baseline function $f_{l}$ and at the same time should not exceed the contrast of the original image, we should restrict $\zeta$ as $1 / r<\zeta \leq 1$ so that the $m \in(1, r]$.

Our contrast measure analysis shows that, for a tone mapping function $f$, if we want to maintain contrast $m$ of $I_{f}$, at the histogram mode $\hat{J}_{l}, f$ should 1) map $\hat{J}_{l}$ to $\hat{J}_{l}$ to preserve the overall image intensity level and 2) should have the tangent slope $m$ at $\hat{J}_{l}$.

Given these two conditions, we insert a anchor point $P=\left(\hat{J}_{l}, \hat{J}_{l}\right)$ with tangent $m$. Recall that the other two anchor points are the endpoints $P^{-}=(0,0)$ and $P^{+}=(1,1)$. Our goal is to construct two Bézier curves, the lower half $B^{-}$from $P^{-}$to $P$ and the upper half $B^{+}$from $P$ to $P^{+}$, to construct $f$. To do so, we introduce two more anchor points $Q^{-}$and $Q^{+}$on the tangent line at $P$ to control the tangent at $P^{-}$and $P^{+}$. Specifically, we can parameterize $Q^{-}$and $Q^{+}$by $\tau^{-}$and $\tau^{+}$:

$$
\begin{aligned}
& Q^{-}=\left(\hat{J}_{l}-\tau^{-} \sin \theta, \hat{J}_{l}-\tau^{-} \cos \theta\right), \\
& Q^{+}=\left(\hat{J}_{l}+\tau^{+} \sin \theta, \hat{J}_{l}+\tau^{+} \cos \theta\right)
\end{aligned}
$$

where $\theta=\arctan (1 / m)$ and $0<\tau^{-} \leq \hat{J}_{l} / \cos \theta$ and 


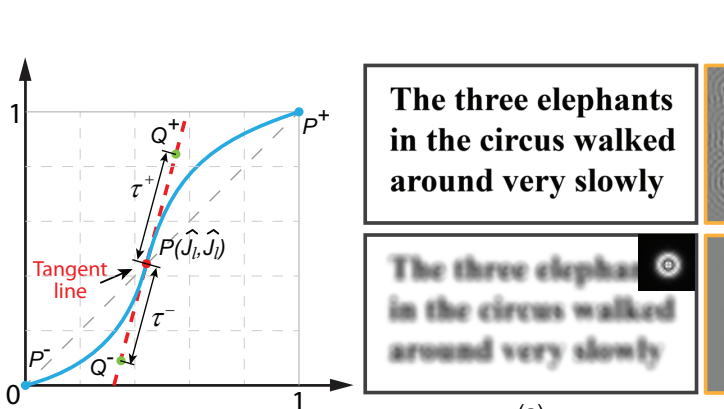

(a)

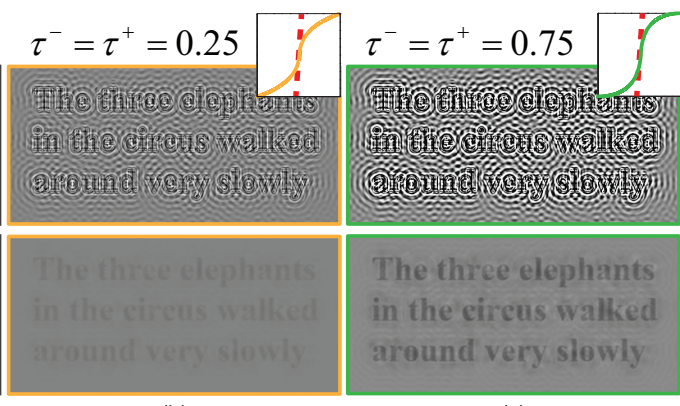

(b)

Figure 4. Left: The construction of an $s$ function. Right: Tone mapping the pre-compensated result using different $s$ functions: (a) The original image and its defocused projection without image pre-compensation; (b) \& (c) are tone mapped and final perceived results by applying different $s$ functions.

$0<\tau^{+} \leq\left(1-\hat{J}_{l}\right) / \cos \theta$. As a result, $P^{-}, Q^{-}$, and $P$ form Bézier curve $B^{-}$and $P, Q^{+}$, and $P^{+}$form Bézier curve $B^{+}$as

$$
\begin{aligned}
& B^{-}(t)=(1-t)^{2} P^{-}+2(1-t) t Q^{-}+t^{2} P \\
& B^{+}(t)=(1-t)^{2} P+2(1-t) t Q^{+}+t^{2} P^{+} \\
& \text {where } \quad 0 \leq t \leq 1
\end{aligned}
$$

By adjusting $\tau^{-}$and $\tau^{+}$and hence $Q^{-}$and $Q^{+}$, we can control the amount of ringing through the curvature of the curves (Section 2.3) while maintaining the desirable contrast, as shown in Fig 4. We call this set of functions the adaptive contrast-priority tone mapping (analogous to shutter/aperture-priority in photography).

Notice that the upper-half Bézier segment $B^{+}$is convex and the lower-half $B^{-}$is concave, forming an " $\mathrm{S}$ " shape. We therefore denote the special mapping function as $s$. Fig. 4 shows the results by using different $s$ functions. The reason that the S-shaped curve $s$ achieves higher contrast factor than the baseline mapping $l$ can also also explained using Jensen's inequality: since the upper-half (the high intensity portion) is convex, by Jensen's inequality we have

$$
I_{s}=B^{+}\left(J_{l}\right) \otimes K>B^{+}\left(J_{l} \otimes K\right)>J_{l} \otimes K=I_{l}
$$

This implies that the brightest pixel in $I_{s}$ will be brighter than the one in the baseline result $I_{l}$. Similarly, since the lower half is concave, the darkest pixel in $I_{s}$ will be dimmer than the one in $I_{l}$. Therefore, the overall dynamic range of final outputs using $s$ will be greater than the one using $l$.

Next, let us compare $s$ with the classical Gamma mapping function. Recall that the Gamma function, i.e., $g(x)=$ $x^{\gamma}$, can be either convex when $\gamma>1$ (denoted as $g^{+}$) or concave when $\gamma<0$ (denoted as $g^{-}$) where both types can be used as the tone mapping function. For $g^{+}$, similar conclusion of Eqn. 10 holds, i.e., the brightest pixel in $I_{g^{+}}$will be brighter than the one in $I_{l}$. However, since $g^{+}$is convex everywhere, the dimmest pixel will also be brighter than the baseline result. Therefore, the overall dynamic range is only marginally expanded. Similar arguments apply to $g^{-}$ which uniformly brings down the intensity for all pixels. Our $s$ function, in contrast, can be viewed as combining the advantage of high intensity potion of $g^{+}$and low intensity portion of $g^{-}$and hence outperforms both.

\subsection{Trading off between Contrast and Ringing}

To balance ringing and contrast, recall that the linear mapping $f_{m}$ produces ringing free result $I_{\mathrm{RF}}$. Therefore, we can set to find the optimal $s$ function that is close to $I_{\mathrm{RF}}$. Specifically, we set out to minimize the following objective function:

$$
\mathcal{O}\left(\tau^{-}, \tau^{+} ; m\right)=\left\|I_{\mathrm{RF}}(m)-I_{s}\left(\tau^{-}, \tau^{+} ; m\right)\right\|+\alpha \frac{1}{m}
$$

The first term measures ringing in terms of the difference between $I_{s}$ and its equivalent ringing-free counterpart $I_{\mathrm{RF}}$. The second term $1 / m$ measures the contrast, i.e., the larger $m(1<m \leq r)$, the higher the contrast. Finally, $\alpha$ is the parameter that trades off between ringing and contrast. A larger $\alpha$ favors more contrast (larger $m$ ) whereas a smaller $\alpha$ favors less ringing (the curve will be closer to linear). We conducted a user study to allow subjects choose their preferred $\alpha$ for specific types of scenes (Section 4.2).

For a given $\alpha$, we use the Levenberg-Marquardt (LM) algorithm [18] to minimize objective function (11). Specifically, we initialize $m$ to its maximum, i.e., $r$ as the initial value, and find $\tau^{-}$and $\tau^{+}$that produce the least ringing by minimizing the first term. We then use the estimated $\tau^{-}$and $\tau^{+}$as initial values to optimize $m$. These steps are iterated to obtain satisfactory results. Fig. 5 shows the optimized results for different $\alpha$.

\section{Applications}

We demonstrate our tone mapping schemes on two image pre-compensation applications.

\subsection{Projection Defocus Compensation}

A projector acts as a camera with an ultra-wide aperture and therefore can only focus at a fixed depth. All projectors suffer from certain blurriness due to imperfect optics 


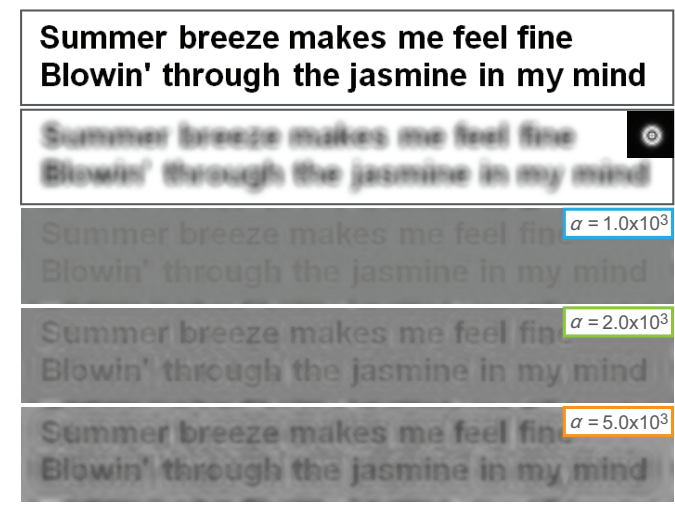

Figure 5. Balance between contrast and ringing. Top two rows show the reference image and the blurred result if we do not precompensate the input. The bottom three rows show the results under different tone mapping functions. A small $\alpha$ loses contrast but incurs minimal ringing. A large $\alpha$ enhances the contrast but incurs more ringing.

or non-planar projection surface. The seminal works of Zhang and Nayar [26] and Brown et al. [2] were the first to compensate defocus blurs using image pre-compensation. Brown et al. [2] and Oyamada and Saito [19] used Wiener filters whereas Grosse et al. [7] later used a coded aperture to improve PSF invertibility. Their techniques work well for small kernels where the dynamic range of the pre-compensated image is about the same as the reference image. Zhang and Nayar [26] bypassed the tone mapping process through constrained iterative steepest descent. At each iteration, they clamp the latest estimation to $[0,1]$. For larger kernels, the optimized results neither guarantee good contrast nor reduced ringing.

Our approach is the first that can actively trade off between contrast and ringing. To validate our approach, we project an Epson Powerlite 3LCD projector out of focus on a wall and captured the projected image using a Canon 60D camera, as shown in Fig. 6. We mount a coded aperture for generating invertible PSFs and project a $36 \times 64$ dot array to estimate the PSF. The reference images $I$ are sharp. For each reference image $I$, we first obtain the pre-compensated image $J$ using Wiener filter and then compute $J_{l}$ (with range $[0,1]$ ). We then compute $J_{l}$ 's histogram and apply our optimization framework to find the optimal contrast-priority tone mapping curve.

Fig. 7 and 8 show two typical examples. Directly using $J_{l}$ as the pre-compensated image significantly loses contrast. Results using steepest descent [26] show enhanced contrast but at the expense of excessive blur. Results produced by our technique appear much sharper, although they also exhibit some ringing. We can use a larger $\alpha$ to reduce ringing at the cost of losing contrast or a smaller $\alpha$ to enhance contrast at the cost incurring more ringing. More results can be found in the supplementary material.

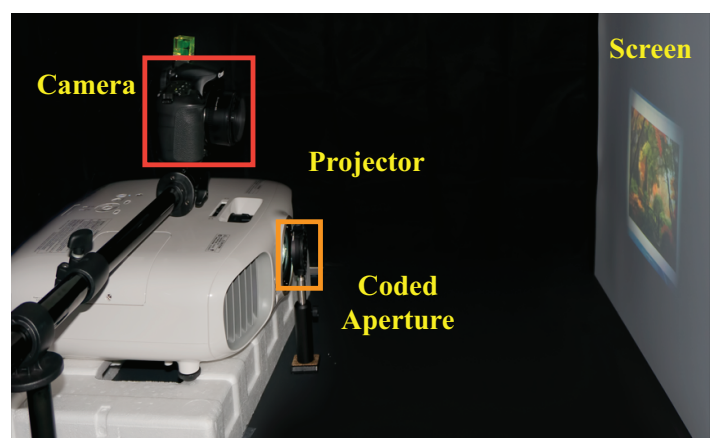

Figure 6. Experimental setup on projector defocus compensation.

\subsection{Improving Visual Acuity}

There is an emerging interest on developing tailored displays for improving visual acuity, e.g., to allow a person with myopia to read without wearing corrective lenses. Recall that both myopia and hyperopia can be viewed as special defocus blurs. Alonso et al. [1] used simple Wiener deconvolution to compute the pre-compensated image and discussed how contrast loss affects the user experience. To enhance contrast, Pamplona et al. [20] designed a special computational display using multiple layers of LCDs. Huang et al. [9] later developed a multi-layer pre-filtering on an ultra-high dynamic range light field display. All these solutions use simple tone mapping functions and rely on the displays to enhance contrast. We show that contrast can be significantly enhanced with a good design of the tone mapping process; our technique is complementary to the computational display approaches.

We emulate the PSFs of myopia/hyperopia using Zernike polynomials [24], which are widely adopted in ophthalmology. The kernel in its radial form is defined as

$$
R_{n}^{m}(\rho)=\sum_{i=0}^{(n-m) / 2} \frac{(-1)^{i}(n-i) !}{i !\left(\frac{1}{2}(n+m)-i\right) !\left(\frac{1}{2}(n-m)-i\right) !} \rho^{n-2 i}
$$

where $0 \leq m \leq n$ and $n-m$ is even. In our experiments, we only consider up to the second order $(n \leq 2)$ terms which can sufficiently model defocus and astigmia. Fig. 10 shows several examples of our PSFs.

We emulate the effect of myopia by convolving sharp text images with the PSFs and display the results on a regular LCD display Viewsonic VA2448 with contrast ratio 1000:1. We then use our algorithm to compute the precompensated images such that after being blurred by the myopia PSFs, will appear readable to the user. In the projector defocus case, the reference image $I$ is generally a natural image with rich color and contrast; here, the reference images are grayscale or even black/white to represent typical texts (e.g., as displayed on the Kindle). Compared with linear tone mapping, our technique significantly enhances the contrast as shown in Fig. 9. 


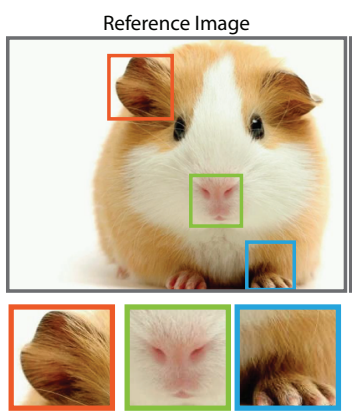

(a)
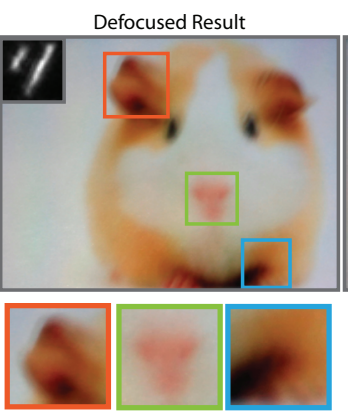

(b)

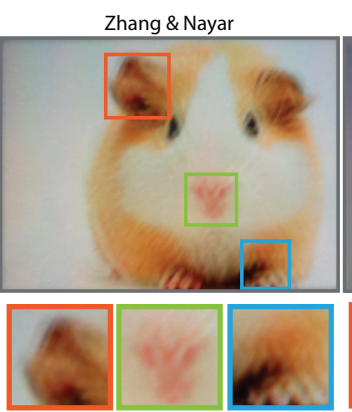

(c)

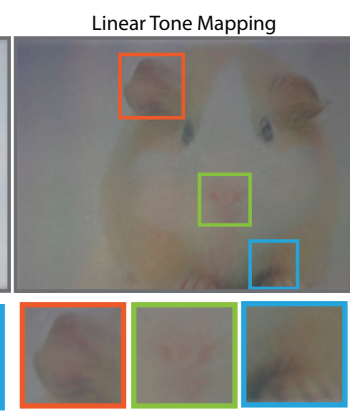

(d)

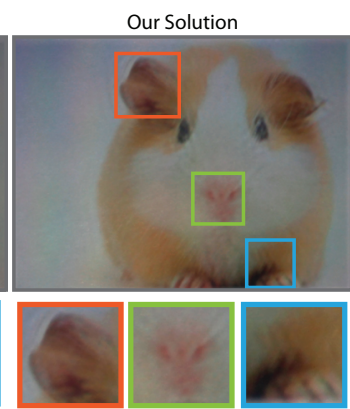

(e)

Figure 7. Defocus compensation on an image. (a) The reference sharp image. (b) The defocused result captured by a Canon 60D. The kernel is shown on the upper-left; (c) Zhang and Nayar's algorithm [26] preserves the contrast but exhibits strong ringing. (d) Linear tone mapping avoids ringing but loses contrast. (e) Our result enhances the contrast with slight ringing.

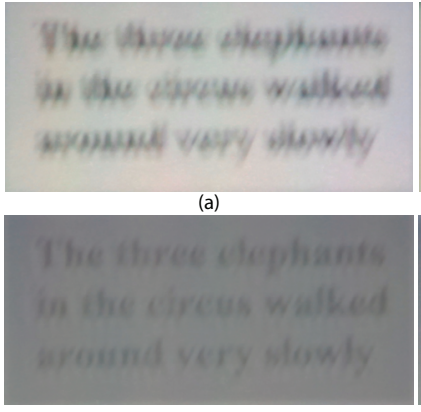

(c)
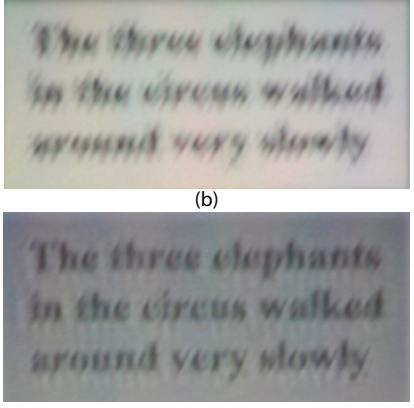

(d)

Figure 8. Defocus compensation on texts. (a) A text image that is defocus blurred (same kernel as Fig. 7). (b) [26] marginally improves the result. (d) Linear tone mapping produces ringingfree result but loses contrast. (e) Our result enhances the contrast with moderate ringing.

User Study Finally, we conduct a simple user study to study users' preference between contrast and ringing. We first develop an interface to allow the user to dynamically change the ringing control factor $\alpha$ in Eqn. 11. We preselect 8 different myopia PSFs and 8 test images (4 natural and 4 text of different font sizes). For every PSF-image pair, the user selects his/her $\alpha$. We have recruited 10 subjects ( 7 male, 3 female), with an average age of 26. Subjects perfor$\mathrm{m}$ the test with corrected vision where myopia is emulated by digitally blurring the image.

Fig. 10 (a) and (b) show the range (in blue) and mean (in red) of $\alpha$ with respect the text images and with respect to the kernels. For a fixed kernel, we find that the range of the preferred $\alpha$ on natural images is much larger than the one on text images. This indicates the heterogeneity of human perception towards the contrast of natural scenes. The users also uniformly prefer a higher contrast even though the displayed images exhibit ringing. In contrast, when viewing text images, the users uniformly prefer a relatively smaller $\alpha$ even though it will sacrifice contrast. This suggests that visual artifacts in texts are more severe to human vision than in natural images. Our results also show that the size of the blur kernels affects the choice of $\alpha$. For smaller kernels

where the loss of contrast is generally low, the divergence of $\alpha$ is high. For larger kernels, the users seem to uniformly prefer low ringing than high contrast if they cannot get both. Further experiments are required to validate our hypotheses and this preliminary studies have already provided some useful insights.

\section{Conclusions and Future Work}

We have presented a new tone mapping scheme for image pre-compensation that can effectively trade between contrast and ringing. We have also carried out in-depth analysis on the causes of dynamic range burst and ringing. Furthermore, we have developed a technique to reliably measure contrast and ringing on images in image pre-compensation. Based on our analysis, we have further designed a contrast-preserving tone mapping function. We have demonstrated our approach in projector defocus compensation and corrective lens free visual enhancement. Compared with the state-of-the-art, our approach not only greatly improves the contrast but also provides an effective interface to trade between contrast and ringing.

Although our user study in visual acuity enhancement illustrates the effectiveness of our solution, it is still primitive. Our immediate future step hence is to first measure the actual myopia/hyperopia PSFs of each individual user, $e . g$., by using the tailored display [20], and use the ground truth PSFs to estimate the tone mapping function. In addition, our Bézier curve model aims to replicate the contrast at the most frequent intensity. If the histogram of the precompensated image has multiple peaks, we can potentially insert multiple anchor points and construct a more complex tone-mapping function. Finally, the problem of contrast preservation can be studied from the perspective of gradients [6]. Our tone mapping function is global and in the future, we plan to explore integrating our contrast model with gradient histogram and gradient domain fusion for handling local contrast enhancements. 


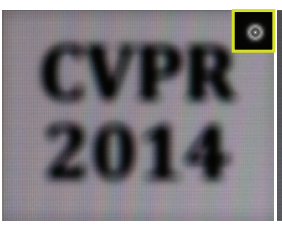

(a)

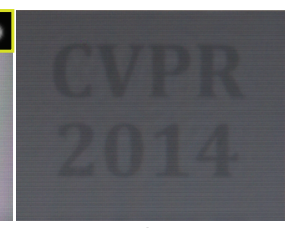

(b)

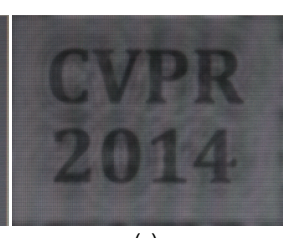

(c)
Figure 9. Emulation of myopia by displaying blurred texts. (a) We use the myopia kernel to blur the text. (b) The result using pre-compensated image under linear tone mapping. (c) The result using our tone mapping function.

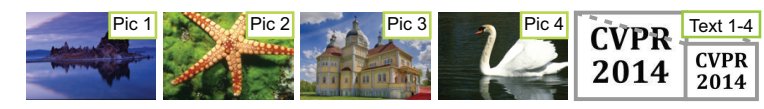

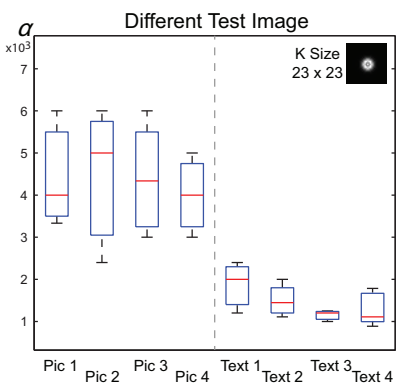

(a)

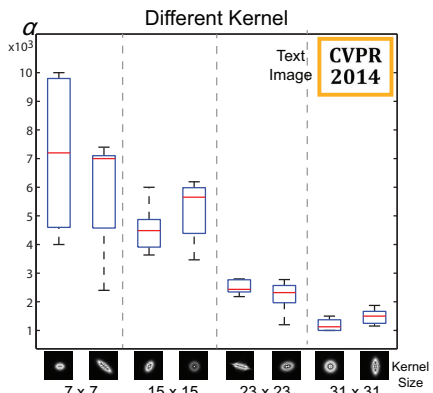

(b)
Figure 10. A user study for evaluating our system. Top: the test images. (a) For a fixed kernel, we plot the range of preferred $\alpha$ across users on different test images. (b) For a fixed test image, we plot the range of preferred $\alpha$ across the users on different kernels. The red bar corresponds to the mean $\alpha$.

\section{Acknowledgements}

This project was supported by the National Science Foundation under grant IIS-CAREER-0845268.

\section{References}

[1] M. Alonso, Jr., A. Barreto, and J. G. Cremades. Image precompensation to facilitate computer access for users with refractive errors. In Proceedings of ACM SIGACCESS conference on Computers and accessibility, 2004.

[2] M. S. Brown, P. Song, and T.-J. Cham. Image preconditioning for out-of-focus projector blur. In CVPR, 2006.

[3] X. Chen, F. Li, J. Yang, and J. Yu. A theoretical analysis of camera response functions in image deblurring. In $E C C V$, 2012.

[4] S. Cho and S. Lee. Fast motion deblurring. In $A C M$ SIGGRAPH Asia), 2009.

[5] H. Farid. Blind inverse gamma correction. IEEE Transactions on Image Processing, 10:1428-1433, Oct 2001.

[6] R. Fattal, D. Lischinski, and M. Werman. Gradient domain high dynamic range compression. ACM Trans. on Graphics (also Proc. of ACM SIGGRAPH), 21(3):249-256, July 2002.

[7] M. Grosse, G. Wetzstein, A. Grundhoefer, and O. Bimber. Coded aperture projection. ACM Trans. on Graphics, 29(3):1-12, 2010.
[8] F.-C. Huang and B. A. Barsky. A frame work for aberration compensated displays. Technical Report UCB/EECS-2011162, University of California, Berkley, Dec 2011.

[9] F.-C. Huang, D. Lanman, B. A. Barsky, and R. Raskar. Correcting for optical aberrations using multilayer displays. ACM Trans. on Graphics (also Proc. of ACM SIGGRAPH Asia), 31, Nov. 2012.

[10] N. Joshi, R. Szeliski, and D. Kriegman. PSF estimation using sharp edge prediction. In $C V P R, 2008$.

[11] N. Joshi, C. L. Zitnick, R. Szeliski, and D. J. Kriegman. Image deblurring and denoising using color priors. In $C V P R$, 2009.

[12] S. Kim, Y.-W. Tai, S. J. Kim, M. Brown, and Y. Matsushita. Nonlinear camera response functions and image deblurring. In $C V P R, 2012$.

[13] D. Krishnan and R. Fergus. Fast image deconvolution using hyper-laplacian priors. NIPS, 22:1-9, 2009.

[14] A. Levin. Blind motion deblurring using image statistics. NIPS, 2006.

[15] A. Levin, R. Fergus, F. Durand, and W. T. Freeman. Image and depth from a conventional camera with a coded aperture. In ACM SIGGRAPH, 2007.

[16] R. Liu, Z. Li, and J. Jia. Image partial blur detection and classification. CVPR, 2008.

[17] D. Martin, C. Fowlkes, D. Tal, and J. Malik. A database of human segmented natural images and its application to evaluating segmentation algorithms and measuring ecological statistics. In Proc. 8th Int'l Conf. Computer Vision, volume 2, pages 416-423, July 2001.

[18] J. Moré. The Levenberg-Marquardt algorithm: Implementation and theory. In Numerical Analysis, Lecture Notes in Mathematics, chapter 10, pages 105-116. Springer Berlin / Heidelberg, 1978.

[19] Y. Oyamada and H. Saito. Focal pre-correction of projected image for deblurring screen image. In $C V P R, 2007$.

[20] V. F. Pamplona, M. M. Oliveira, D. G. Aliaga, and R. Raskar. Tailored displays to compensate for visual aberrations. ACM Trans. on Graphics, 31(4):81:1-81:12, July 2012.

[21] E. Peli. Contrast in complex images. J. Opt. Soc. Am. A, 7(10):2032-2040, Oct 1990.

[22] J. G. Proakis and D. G. Manolakis. Digital signal processing. Pearson Prentice Hall, NJ, USA, 4 edition, 2006.

[23] Y.-W. Tai, X. Chen, S. Kim, S. J. Kim, F. Li, J. Yang, J. Yu, Y. Matsushita, and M. S. Brown. Nonlinear camera response functions and image deblurring: Theoretical analysis and practice. IEEE Transactions on Pattern Analysis and Machine Intelligence, 35(10):2498-2512, 2013.

[24] J. Y. Wang and D. E. Silva. Wave-front interpretation with zernike polynomials. Appl. Opt., 19(9):1510-1518, May 1980.

[25] Y. Yitzhaky, I. Mor, A. Lantzman, and N. S. Kopeika. A direct method for restoration of motion blurred images. Journal of the Optical Society of America A: Optics, Image Science, and Vision, 15:1512-1519, 1998.

[26] L. Zhang and S. K. Nayar. Projection Defocus Analysis for Scene Capture and Image Display. ACM Trans. on Graphics (also Proc. of ACM SIGGRAPH), Jul 2006. 